\title{
Acupoints Stimulation for Anxiety and Depression in Cancer Patients: A Quantitative Synthesis of Randomized Controlled Trials
}

\author{
Tao Wang, ${ }^{1}$ Renli Deng, ${ }^{1}$ Jing-Yu Tan, ${ }^{2}$ and Feng-Guang Guan ${ }^{3}$ \\ ${ }^{1}$ The Fifth Affiliated (Zhuhai) Hospital of Zunyi Medical University, No. 1439, Zhufeng Road, Zhuhai, Guangdong 519100, China \\ ${ }^{2}$ School of Nursing, Fujian University of Traditional Chinese Medicine, No. 1, Qiuyang Road, Fuzhou, Fujian 350122, China \\ ${ }^{3}$ The Second Affiliated People's Hospital, Fujian University of Traditional Chinese Medicine, \\ No. 282, Wusi Road, Fuzhou, Fujian 350003, China \\ Correspondence should be addressed to Renli Deng; renli.deng@gmail.com and Feng-Guang Guan; fg_guan@hotmail.com
}

Received 13 June 2015; Revised 9 November 2015; Accepted 17 November 2015

Academic Editor: Mary K. Garcia

Copyright (C) 2016 Tao Wang et al. This is an open access article distributed under the Creative Commons Attribution License, which permits unrestricted use, distribution, and reproduction in any medium, provided the original work is properly cited.

\begin{abstract}
This study aims at concluding the current evidence on the therapeutic effects of acupoints stimulation for cancer patients with anxiety and depression. Randomized controlled trials using acupoints stimulation for relieving anxiety and/or depression in cancer patients were searched, and 11 studies were finally included, of which eight trials compared acupoints stimulation with standard methods of treatment/care, and acupoints stimulation showed significantly better effects in improving depression than using standard methods of treatment/care. Four studies compared true acupoints stimulation with sham methods, and no significant differences can be found between groups for either depression or anxiety, although the pooled effects still favored true intervention. For the five studies that evaluated sleep quality, the results were conflicting, with three supporting the superiority of acupoints stimulation in improving sleep quality and two demonstrating no differences across groups. Acupoints stimulation seems to be an effective approach in relieving depression and anxiety in cancer patients, and placebo effects may partially contribute to the benefits. However, the evidence is not conclusive due to the limited number of included studies and the clinical heterogeneity identified among trials. More rigorous designed randomized, sham-controlled studies are necessary in future research.
\end{abstract}

\section{Introduction}

Cancer is one of the major health problems in the world. The incidence of cancer and the related death have significantly increased during the past decades. According to the global corresponding data reported by the International Agency for Research on Cancer (IARC), 14.1 million cancer patients were identified around the world and 8.2 million patients died in 2012 [1]. Cancer-related death has gradually surpassed the relative figure of all types of heart disease and stroke and has become the leading cause of death at present [2].

With the advance of medical technologies, cancer treatments have changed reasonably, and comprehensive treatment strategies including optimized pharmacological intervention [3] and palliative care can ease most physical symptoms effectively and let cancer survivors live a relatively symptom-free life [4, 5]. Although the survival rate has been rising over the past decade, the negative cancer experiences and the side effects associated with cancer treatments (e.g., pain, fatigue, nausea, and vomiting) can result in a wide range of health problems, which contain not only physical issues but also psychological and emotional distresses including depression, anxiety, and posttraumatic stress disorder (PTSD) [59]. The incidence of depression in cancer survivors is three to four times as much as that in general population [10], ranging from $10 \%$ to $50 \%[11,12]$. The percentages of anxiety and PTSD following cancer were reported to be $6 \%$ to $23 \%$ [13] and $0 \%$ to $32 \%$ [14], respectively. The negative emotional feelings can contribute to significantly undesirable impacts on cancer patients, leading to sleep disturbance, loss of appetite, deterioration of quality of life, and so forth [15]. In turn, the psychological and emotional disorders can 
also increase the risk of recurrence and mortality of cancer as psychological distresses could impair patients' immune response [16].

Healthcare professionals have recognized that cancer treatment should aim at not only expanding patients' life span but also improving their psychosocial well-being [17]. In recent years, healthcare professionals and patients are increasingly interested in using complementary therapies to relieve cancer survivors' emotional problems such as anxiety and depression and make them as alternatives to the mainstream medicine [18]. Acupoints stimulation as one kind of complementary therapies originates from ancient China, and it is based on the theory of main and collateral channels. For the modalities of acupoints stimulation, both acupuncture and acupressure are used in practice. The World Health Organization (WHO) has viewed acupoints stimulation as a beneficial intervention to deal with a wide range of health problems [19], and its therapeutic effects for psychological distresses, particularly with regard to anxiety and depression, have been explored in noncancer populations, and corresponding systematic reviews and clinical trials have been conducted to identify its positive effects [20-23]. Cancer patients as the special population suffer more from anxiety and depression [10], and acupoints stimulation can be a promising therapy, but the evidence remains unknown.

Preclinical research has indicated that both the hypothalamic-pituitary-gonadal axis (HPA) and the sympathetic nervous system (SNS) can be significantly affected by acupoints stimulation, which presents a possible explanation that acupoints stimulation may have a positive impact on reducing psychological distresses from the biological perspectives [24, 25]. A large number of clinical trials have also been conducted to explore whether acupoints stimulation is effective in controlling anxiety and depression in cancer patients but their findings were conflicting [26-36]. Some demonstrated that acupoints stimulation can effectively relieve anxiety and/or depression while some did not. The clinical heterogeneity in patient characteristics, intervention protocols, and outcome variables and the various degrees of methodological quality among those trials may partially contribute to the contradictory study results. However, to our knowledge, currently there is no systematic summary in regard to the methodological quality assessment of the trials on acupoints stimulation for anxiety and depression in cancer patients, and the overall evidence on its clinical efficacy remains uncertain. Therefore, the objectives of this systematic review are to explore the current evidence on acupoints stimulation for anxiety and depression in cancer patients and to offer recommendations for future research and practice.

\section{Methods}

2.1. Search Strategies. We retrieved relevant references by searching electronic databases. Ten databases were searched (up to November 20, 2014) which include PubMed, Cochrane Central Register of Controlled Trials (CENTRAL), Cumulative Index to Nursing and Allied Health Literature (CINAHL), Allied and Complementary Medicine (AMED),
PsycINFO, ISI Web of Science, Science Direct, WanFang Data, China National Knowledge Infrastructure (CNKI), and Chinese Scientific Journal Database (VIP). When conducting the electronic search, we did not set restrictions for the publications in terms of types of language and types of study. Mesh terms and key words used in the search strategies were "acupuncture", "acupressure", "acupuncture, ear", "electroacupuncture", "neoplasms", "anxiety", "depression", "mood disorders", and so forth; we also searched the references of the final included articles and other Chinese core journals on Traditional Chinese Medicine (TCM) to see whether we can locate any publications for possible inclusion. The literature search was conducted independently by two reviewers. In Table 4, we list two search strategies for this systematic review.

2.2. Study Selection and Data Extraction. After completing literature search, the same two reviewers independently screened the title and abstract of all searched articles, and the final eligible articles were obtained and read in full text. Inclusion criteria for eligible studies were (1) randomized controlled trials comparing acupoints stimulation (including manual acupuncture, electroacupuncture, or acupressure) to one or more of the following situations: sham acupoints stimulation, standard treatment/care (usual care, standard medication, and attention control group were all defined as standard treatment/care in this systematic review), or waitlist control; (2) participants who should be cancer patients with anxiety and/or depression regardless of being children or adults; and (3) trials published in English or Chinese.

The primary outcome measures were anxiety and depression as measured by Hospital Anxiety and Depression Scale (HADS), Center for Epidemiologic Studies Depression Scale (CESD), Hamilton Depression Scale (HAMD), Beck Depression Inventory-Primary Care (BDI-PC), and so forth, while the secondary outcome was the status of sleep quality as measured by Pittsburgh Sleep Quality Index (PSQI), sleep $\log$, and so forth. Characteristics of the included studies were extracted through a data extraction form which was piloted prior to the commencement of the study.

2.3. Methodological Quality Assessment for the Included Studies. We evaluated the methodological quality of each included trial with the 2015 risk of bias criteria provided by the Cochrane Back and Neck (CBN) Group [37], which include 13 specific domains: random sequence generation, allocation concealment, blinding of participants, blinding of caregiver, blinding of outcome assessor, description and acceptance of dropout rate, intention-to-treat analysis, selective outcome reporting, baseline similarity, cointerventions, acceptable compliance, timing of outcome assessment, and other sources of bias (e.g., validity of the outcome assessments and the report of the conflict of interest). The risk of bias criteria used in this study are adapted from the Cochrane Handbook of Reviews of Interventions and are appropriate for studies using nonpharmacological intervention [37]. Two reviewers independently assessed the risk of bias of the included RCTs. Each of the 13 risk of bias items was rated as "low risk of bias," "unclear risk of bias," or "high risk of bias." 


\begin{tabular}{|c|c|}
\hline \multicolumn{2}{|c|}{$\begin{array}{l}\text { Records identified through search of databases and other sources }(N=1135) \\
\text { PubMed }(n=251) \text {, CENTRAL }(n=84) \text {, CINAHL }(n=95) \text {, AMED }(n=45) \text {, PsycINFO }(n=56) \text {, } \\
\text { Web of Science }(n=493) \text {, Science Direct }(n=97) \text {, WanFang }(n=3) \text {, CNKI }(n=4) \text {, and VIP }(n=7)\end{array}$} \\
\hline & Duplicated records $(N=254)$ \\
\hline \multicolumn{2}{|c|}{ Browsing title and abstract of each record $(N=881)$} \\
\hline & Records excluded $(N=817)$ \\
\hline \multicolumn{2}{|c|}{ Full-text articles evaluated for eligibility $(N=64)$} \\
\hline & $\begin{array}{l}\text { Full-text articles excluded }(N=53) \\
\text { Review paper }(n=4) \\
\text { Clinical case report }(n=3) \\
\text { Qualitative study }(n=2) \\
\text { Nonrandomized controlled trial }(n=7) \\
\text { Participants not meeting the inclusion criteria }(n=4) \\
\text { Interventions not meeting the inclusion criteria }(n=8) \\
\text { Duplications }(n=6) \\
\text { Measures not meeting the inclusion criteria }(n=18) \\
\text { Study published in Korean }(n=1)\end{array}$ \\
\hline \multicolumn{2}{|c|}{$\begin{array}{l}\text { Articles included for the systematic review }(N=11) \\
\text { PubMed }(n=4) \text {, CENTRAL }(n=5) \text {, CINAHL }(n=0) \text {, AMED }(n=0) \text {, PsycINFO }(n=0) \text {, } \\
\text { Web of Science }(n=0) \text {, Science Direct }(n=0) \text {, WanFang }(n=1) \text {, CNKI }(n=0) \text {, and VIP }(n=1)\end{array}$} \\
\hline
\end{tabular}

FIGURE 1: Flow chart of study selection.

Methodological quality assessment was further checked by a third review author and any disagreements on the risk of bias judgment were handled by discussion.

2.4. Data Analysis. We used the Review Manager 5.3 (Cochrane Collaboration) to conduct the data synthesis. Regarding the continuous outcome variables, we calculated the standardized mean difference (SMD) or the weighted mean difference (WMD) with $95 \%$ confidence intervals (CI). Fixed effect model was considered when the trials had a satisfactory statistical homogeneity which was evaluated by examining $I^{2}\left(I^{2}<50 \%\right)$. Otherwise, a random effect model was applied. Both overall assessment and subgroup estimation were performed. The overall assessment on the effects of acupoints stimulation for mood problems and sleep quality was performed first regardless of the acupoints stimulation types. Subgroup analysis based on different acupoints stimulation modalities (manual acupuncture, electroacupuncture, and acupressure) was carried out afterwards. If the data synthesis could not be conducted owing to the absence of available data, different types of data, or a significant heterogeneity in the outcome effect, descriptive analysis was considered instead.

\section{Results}

3.1. Characteristics of the Included Studies. A total of 1135 potential records were yielded by searching the electronic databases, and 11 trials [26-36] were finally retrieved for systematic review. The flow chart (Figure 1) presents the selection process of the qualified studies. The 11 included studies, with nine English and two Chinese articles, were all journal papers published between 2006 and 2014. The study sample ranged from 30 to 302 and the average sample size was 98. A total of 1073 participants (number of randomized participants) with various types of malignancies were involved. Six studies focused on breast cancer [26, 29, 31-34], one study was on lung cancer [30], one study was on gynecological cancer [36], and three studies [27, 28, 35] involved more than two types of malignancies. For the study design, eight [26$28,31-33,35,36]$ were two-arm design and the other three $[29,30,34]$ studies included three study arms.

Anxiety and/or depression were assessed in 11 studies. Seven of them assessed anxiety, out of which six studies $[26,27,29,30,33,34]$ used Hospital Anxiety and Depression Scale (HADS-A) which is commonly used in clinical research related to emotional problems in cancer [38] and the other one [32] adopted Psychological and General Well-Being Index (PGWB) which is a validated quality of life (QoL) instrument and "anxiety" is included as one of the subscales. Eleven studies assessed depression, out of which one study [26] employed CESD, one [36] applied HAMD only, two $[28,35]$ used both HAMD and Self-Rating Depression Scale (SDS) (only data from HAMD was used for data synthesis in order to ensure the homogeneity of outcome measures among trials), five $[27,29,30,33,34]$ employed HADS subscale for depression (HADS-D) only, and two studies adopted BDIPC [31] and Psychological and General Well-Being Index 
(PGWB) [32], respectively. Sleep quality was assessed in five studies $[26,28-30,32]$ and the PSQI was employed in four trials [26, 28-30], while in the other one study [32], sleep quality was measured by recording the times of wake-up and the hours of sleep on log book. Characteristics of the included studies are presented in Table 1.

3.2. Description of the Intervention Protocol. Intervention protocols used in the included studies are also summarized in Table 1. Three acupoints stimulation modalities were employed, of which seven [26-28, 31, 33, 34, 36] adopted manual acupuncture and three [29, 32, 35] used electroacupuncture while only one trial [30] chose acupressure. Of the seven studies that adopted manual acupuncture, two $[26,27]$ assessed its therapeutic effects by comparing true acupuncture with sham acupuncture (selected sham acupoints were located away from the true acupoints), four studies [28, 31, 33, 36] compared true acupuncture with standard treatment/care (two [28, 31] adopted standard medication, specifically antidepressant, as the standard treatment and the remaining two $[33,36]$ used attention control or usual care), and one trial [34] with three-arm design investigated the effects of acupuncture by comparing the true acupuncture which was conducted by acupuncture practitioners with two types of controls: the self-acupuncture control conducted by the participants and the standard treatment/care control without acupuncture. Of the three studies that used electroacupuncture, one compared true electroacupuncture with either sham electrostimulation or wait-list control [29] and the other two studies $[32,35]$ compared true electroacupuncture with standard treatment/care (standard medication in one study [32] and usual care in the other [35]). The only one study [30] employing acupressure was a three-arm design, with one group using acupressure plus oil and the other two adopting true acupressure and sham intervention, respectively. For this study, we only abstracted the data from the true and sham acupressure groups for analysis. Ten studies described the protocols of acupoints stimulation, which included the selection and identification of targeted acupoints, types of stimulation, and duration and frequency of treatment. Regarding the selected acupoints, Sanyinjiao (SP6), Zusanli (ST36), and Hegu (LI4), which can be identified in nearly half of the included studies, were the most frequently adopted acupoints for depression and/or anxiety.

3.3. Methodological Quality and Risk of Bias of the Included Trials. Table 2 shows the results of the risk of bias assessment for the included studies. Methodological quality of the trials was generally acceptable. All of the analyzed trials mentioned the method of randomization, and details of producing the random sequence were reported in nine studies by applying random number table, using computer software, or tossing coin. Treatment allocation was reported to be concealed in only five studies. Blinding of participants, care provider, or outcome assessor was performed in four studies. All studies reported the dropout of study subjects and in ten of them the dropout rates were deemed as acceptable (less than 20\%) [37], whereas, in the other one study, the dropout rate exceeded $30 \%$ in the long-term observation (high risk of bias). In eight trials, all subjects with randomization were included for data analysis based on missing data handling approach such as intention-to-treat analysis. Only one study [31] failed to specify whether it had selectively reported study outcomes or not. All studies were similar at baseline regarding the major demographic variables, and the timing of the outcome assessment was also reported to be similar in all the included trials.

3.4. Therapeutic Effects of Acupoints Stimulation. Results of the data synthesis for the systematic review are summarized in Table 3.

\subsubsection{Overall Assessment of Acupoints Stimulation for Anxiety and Depression}

(i) Acupoints Stimulation versus Standard Methods of Treatment/Care. Eight studies [28, 29, 31-36] compared the effects of acupoints stimulation with standard methods of treatment/care. All of the eight trials evaluated depression, four $[29,32-34]$ measured anxiety, and three $[28,29,32]$ measured sleep quality. Meta-analysis indicated that acupoints stimulation can significantly relieve depression in cancer patients [random effect model, SMD $=-1.08,95 \%$ CI $=-1.97$ to -0.19 , and $P=0.02]$. However, statistical heterogeneity $\left(P<0.0001, I^{2}=94 \%\right)$ was identified in the pooled effects, and removal of any suspicious trials did not change the heterogeneity significantly. For the eight studies that evaluated depression, two trials [28, 31] compared acupuncture with standard medication (antidepressant), and data synthesis was not conducted due to different kinds of outcome measures. According to the descriptive analysis of each single trial, acupoints stimulation (acupuncture) demonstrated a similar [31] or even better effect [28] in controlling depression than using antidepressant.

Data synthesis cannot be conducted for anxiety due to the insufficient number of studies and the inconsistent types of data. Descriptive analysis was adopted instead. Two trials $[29,33]$ showed significant improvements in anxiety. In the study conducted by Mao et al. [29], acupoints stimulation (electroacupuncture) was found to be effective in relieving anxiety in cancer survivors $(P=0.044)$ after ten treatments over eight weeks. There are two articles $[33,34]$ reporting two different study phases from one large trial. In one paper published in 2012 [33], a 6-week acupuncture treatment led by the acupuncturists was compared with enhanced usual care (attention control) which was implemented by providing patients with an information booklet to maintain the consistency of the control group, and the results favored acupuncture in reducing anxiety $(P<0.01)$. Sample from the acupuncture group was rerandomized in a further trial [34] to compare the effects of 4-week acupuncturist-delivered acupuncture with either 4-week self-acupuncture or no maintenance group (usual care), and no marked differences in anxiety scores were detected across groups.

For sleep quality, conflicting study results were reported where one study [28] favored acupoints stimulation (acupuncture) in improving the quality of sleep $(P<0.01)$, while another one [29] reported that acupoints stimulation 


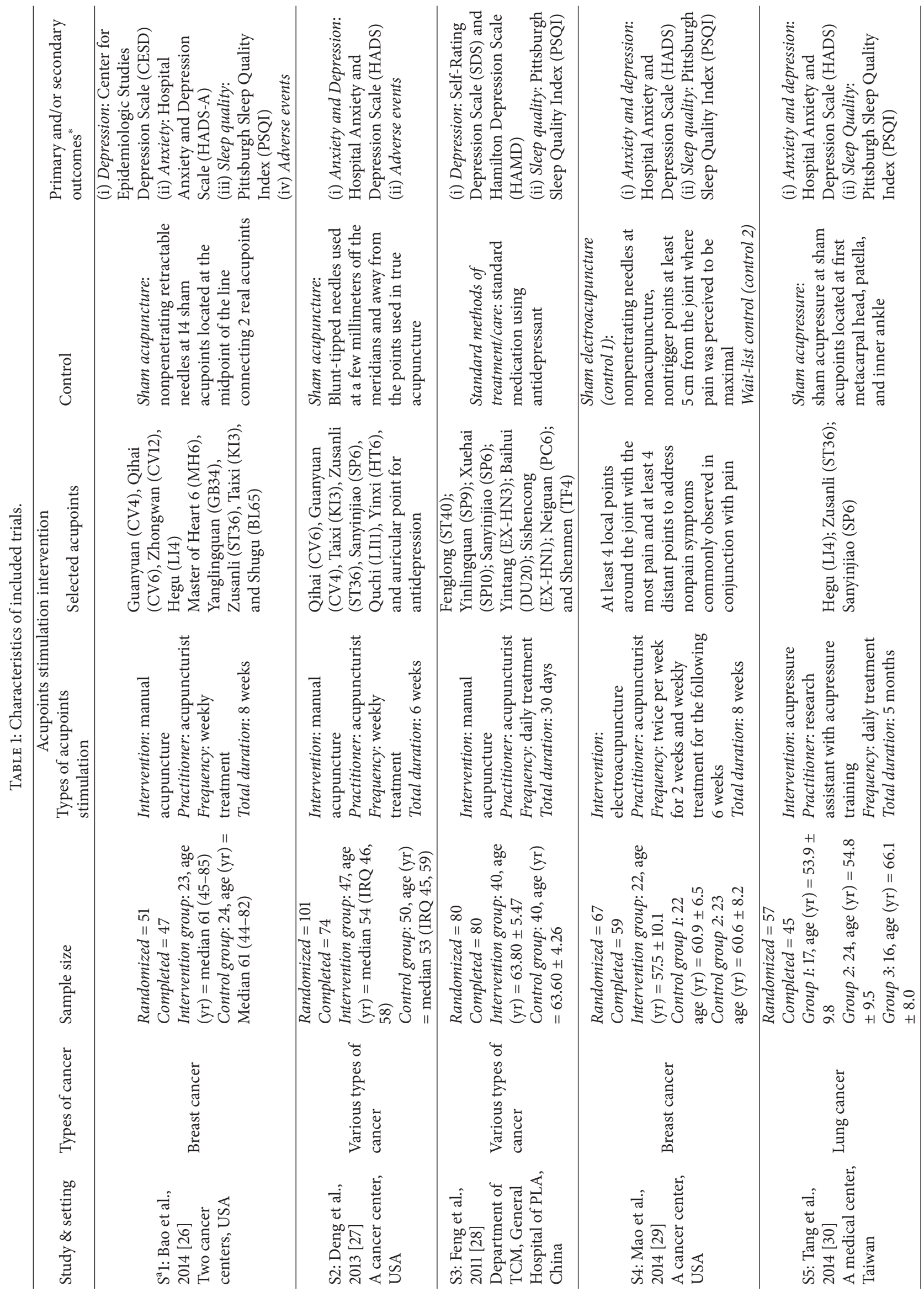




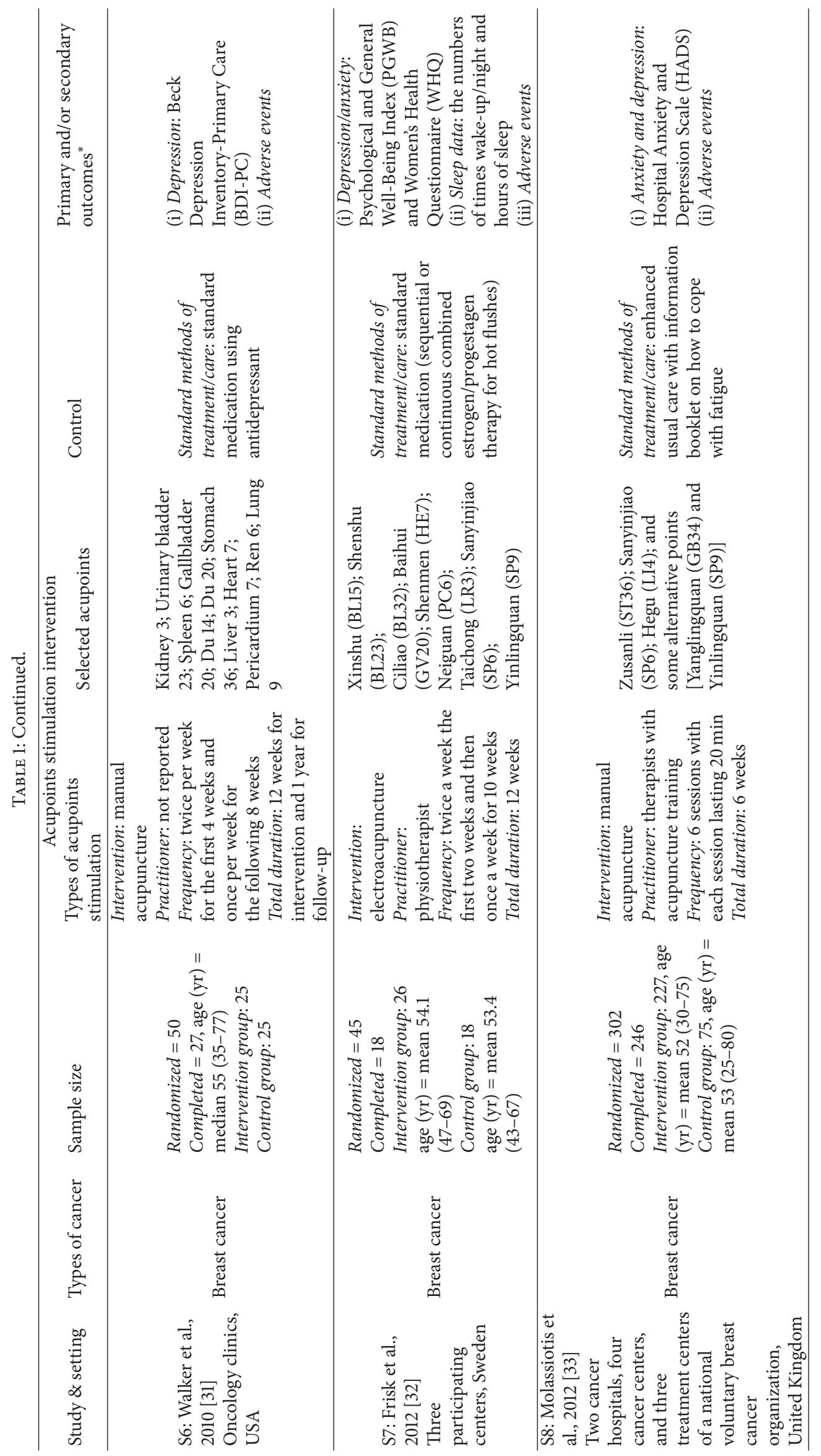




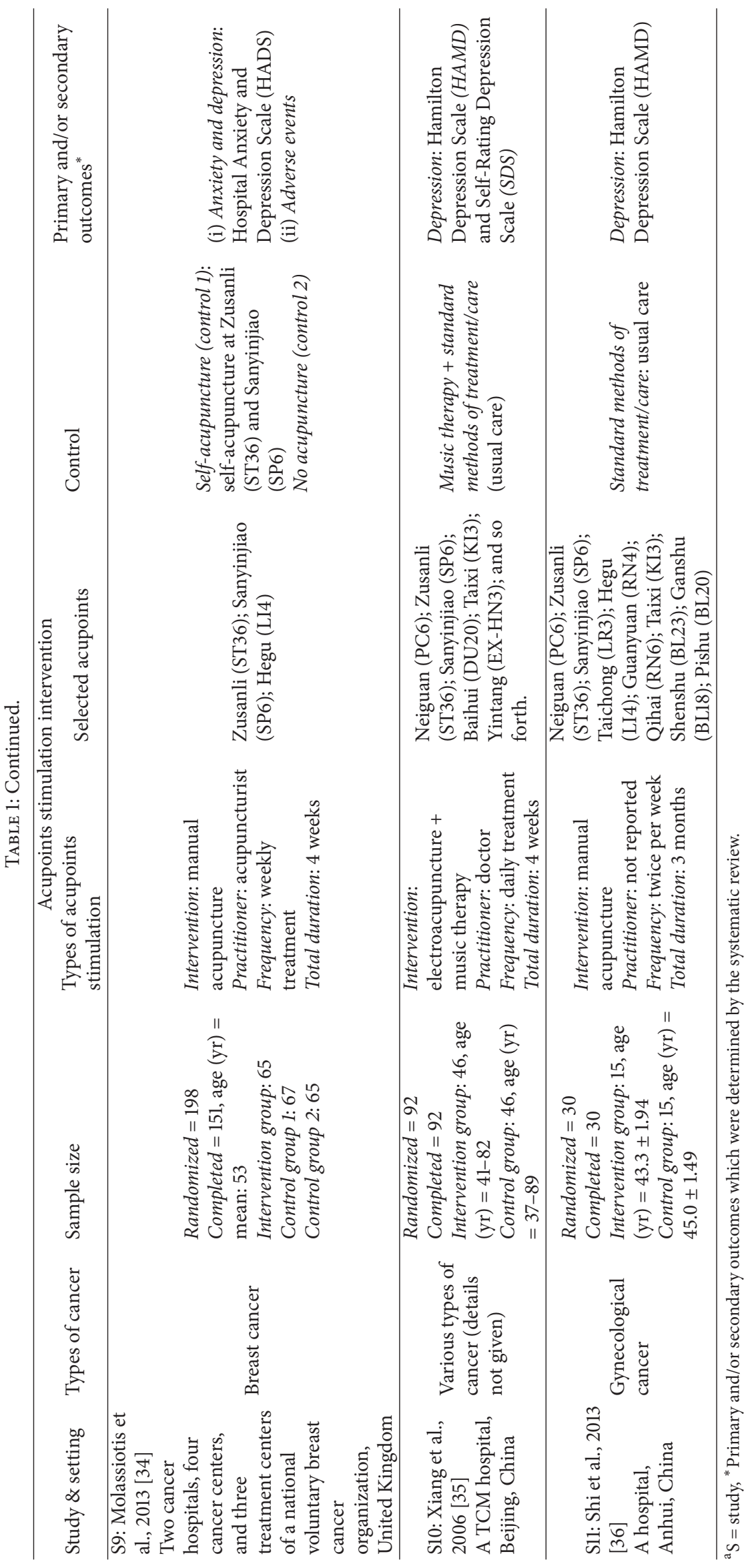


TABLE 2: Methodological and quality assessment of included trials.

\begin{tabular}{|c|c|c|c|c|c|c|c|c|c|c|c|}
\hline Criteria & $\mathrm{S}^{\mathrm{a}} 1$ & S2 & S3 & S4 & S5 & S6 & S7 & S8 & S9 & S10 & S11 \\
\hline $\begin{array}{l}\text { (1) "Was the method of } \\
\text { randomization adequate?" }\end{array}$ & $r$ & $\checkmark$ & $\checkmark$ & $r$ & $\checkmark$ & $?$ & $r$ & $r$ & $r$ & $\checkmark$ & $?$ \\
\hline $\begin{array}{l}\text { (2) "Was the treatment } \\
\text { allocation concealed?" }\end{array}$ & $x$ & $r$ & $?$ & $r$ & $?$ & $?$ & $r$ & $r$ & $r$ & $?$ & $?$ \\
\hline $\begin{array}{l}\text { (3) "Was the patient blinded to } \\
\text { the intervention?" }\end{array}$ & $r$ & $r$ & $?$ & $r$ & $r$ & $?$ & $?$ & $x$ & $x$ & $?$ & $?$ \\
\hline $\begin{array}{l}\text { (4) "Was the care provider } \\
\text { blinded to the intervention?" }\end{array}$ & $x$ & $x$ & $?$ & $x$ & $x$ & $?$ & $?$ & $x$ & $x$ & $?$ & $?$ \\
\hline $\begin{array}{l}\text { (5) "Was the outcome assessor } \\
\text { blinded to the intervention?" }\end{array}$ & $r$ & $r$ & $?$ & $r$ & $r$ & $?$ & $?$ & $x$ & $x$ & $?$ & $?$ \\
\hline $\begin{array}{l}\text { (6) "Was the drop-out rate } \\
\text { described and acceptable?" }\end{array}$ & $r$ & $r$ & $r$ & $r$ & $r$ & $r$ & $x$ & $r$ & $r$ & $r$ & $\checkmark$ \\
\hline $\begin{array}{l}\text { (7) "Were all randomized } \\
\text { participants analyzed in the } \\
\text { group to which they were } \\
\text { allocated?" }\end{array}$ & $r$ & $r$ & $r$ & $r$ & $r$ & $r$ & $x$ & $x$ & $x$ & $r$ & $\checkmark$ \\
\hline $\begin{array}{l}\text { (8) "Are reports of the study } \\
\text { free of suggestion of selective } \\
\text { outcome reporting?" }\end{array}$ & $r$ & $\checkmark$ & $r$ & $r$ & $r$ & $?$ & $r$ & $r$ & $r$ & $r$ & $\checkmark$ \\
\hline $\begin{array}{l}\text { (9) "Were the groups similar } \\
\text { at baseline regarding the most } \\
\text { important prognostic } \\
\text { indicators?" }\end{array}$ & $\checkmark$ & $r$ & $\checkmark$ & $\checkmark$ & $r$ & $r$ & $r$ & $r$ & $r$ & $\checkmark$ & $r$ \\
\hline $\begin{array}{l}\text { (10) "Were co-interventions } \\
\text { avoided or similar?" }\end{array}$ & $\checkmark$ & $\checkmark$ & $?$ & $r$ & $?$ & $?$ & $r$ & $?$ & $?$ & $r$ & $?$ \\
\hline $\begin{array}{l}\text { (11) Was the compliance } \\
\text { acceptable in all groups? }\end{array}$ & $?$ & $?$ & $?$ & $r$ & $r$ & $?$ & $\checkmark$ & $r$ & $\checkmark$ & $?$ & $?$ \\
\hline $\begin{array}{l}\text { (12) "Was the timing of the } \\
\text { outcome assessment similar in } \\
\text { all groups?" }\end{array}$ & $\checkmark$ & $r$ & $r$ & $\checkmark$ & $r$ & $\checkmark$ & $\checkmark$ & $r$ & $r$ & $\checkmark$ & $r$ \\
\hline $\begin{array}{l}\text { (13) "Are other sources of } \\
\text { potential bias unlikely?" }\end{array}$ & $?$ & $?$ & $?$ & $?$ & $?$ & $?$ & $?$ & $?$ & $?$ & $?$ & $?$ \\
\hline
\end{tabular}

Based on [37].

${ }^{\mathrm{a}} \mathrm{S}$ : study, $\checkmark$ : low risk of bias; $X$ : high risk of bias; ?: unclear risk of bias.

(electroacupuncture) failed to contribute to significant improvement in PSQI score $(P=0.058)$ when comparing with the wait-list control.

(ii) Acupoints Stimulation versus Sham Acupoints Stimulation. Acupoints stimulation was compared with sham intervention in four trials [26, 27, 29, 30]. All of them involved anxiety and depression and three $[26,29,30]$ assessed sleep quality. For anxiety and depression, only two studies were eligible for synthesis. Although the differences between groups did not reach a statistical significance, the pooled effects still favored true acupoints stimulation in relieving either anxiety [random effect model, $\mathrm{MD}=-0.65,95 \% \mathrm{CI}=-2.84$ to 1.53 , and $P=0.56$ ] or depression [random effect model, $\mathrm{MD}=-0.27,95 \% \mathrm{CI}=-2.66$ to 2.12 , and $P=0.82]$. For sleep quality, descriptive analysis from one trial [30] indicated that acupoints stimulation (acupressure) could improve sleep quality $(P=0.040)$.

\subsubsection{Subgroup Analysis on Manual Acupuncture}

(i) Manual Acupuncture versus Standard Methods of Treatment/Care. Manual acupuncture on anxiety and/or depression was compared with standard methods of treatment/care in five studies [28, 31, 33, 34, 36], and manual acupuncture was found to be superior in depression relief [random effect model, $\mathrm{MD}=-2.55,95 \% \mathrm{CI}=-3.61$ to -1.49 , and $P<$ $0.00001]$. However, significantly statistical heterogeneity $(P=$ $0.002, I^{2}=80 \%$ ) was identified in the pooled effect. This might be caused by Feng et al.'s study [28] as the $I^{2}$ decreased to $0 \%(P=0.89)$ after removing the mentioned trial, and the pooled effect was relatively stable, which still favored manual acupuncture [random effect model, $\mathrm{MD}=-2.10,95 \%$ $\mathrm{CI}=-2.56$ to -1.64 , and $P<0.00001]$. Descriptive analysis also supported the superiority of acupuncture in managing anxiety $(P<0.01)[33]$ and improving sleep quality $(P<$ $0.01)[28]$ in cancer patients. 


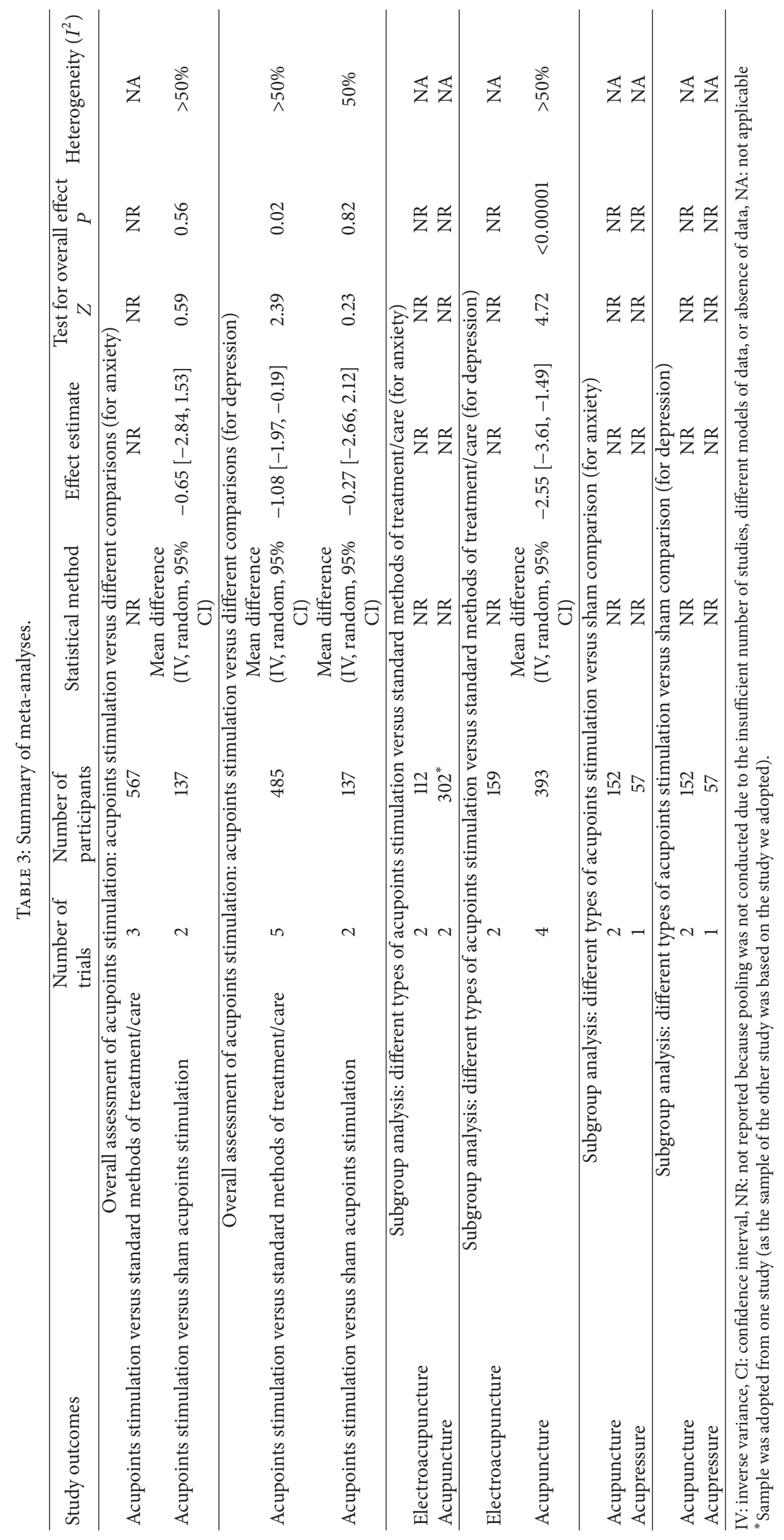


TABLE 4: Selected search strategies.

\begin{tabular}{|c|c|c|}
\hline ID & Searching strategies & Records \\
\hline \multicolumn{3}{|c|}{ PubMed } \\
\hline$\# 1$ & Search “acupuncture”[MeSH Terms] & 17708 \\
\hline$\# 2$ & Search “acupuncture therapy”[MeSH Terms] & 16944 \\
\hline$\# 3$ & Search “acupressure”[MeSH Terms] & 465 \\
\hline$\# 4$ & Search “acupuncture, ear”[MeSH Terms] & 267 \\
\hline$\# 5$ & Search “acupuncture points”[MeSH Terms] & 4063 \\
\hline \#6 & Search "electroacupuncture"[MeSH Terms] & 2566 \\
\hline$\# 7$ & Search “auriculotherapy”[MeSH Terms] & 277 \\
\hline$\# 8$ & $\# 1$ OR \#2 OR \#3 OR \#4 OR \#5 OR \#6 OR \#7 & 17958 \\
\hline \#9 & $\begin{array}{l}\text { Search }\left(\left(\left(\left(\left(\left(\left(\text { acupunctur* }{ }^{*} \text { Title/Abstract] }\right) \text { OR acupressure* [Title/Abstract] }\right) \text { OR }\right.\right.\right.\right.\right. \\
\text { auriculotherap* [Title/Abstract] }) \text { OR electroacupunctur* [Title/Abstract] }) \text { OR acupoint }{ }^{*}[\text { Title/Abstract] }) \text { OR } \\
\text { needl* [Title/Abstract] }) \text { OR acupuncture therapy[Title/Abstract] }) \text { OR "ear acupuncture"[Title/Abstract] }) \text { OR } \\
\text { acupuncture points[Title/Abstract] }\end{array}$ & 104918 \\
\hline$\# 10$ & $\# 8$ or \#9 & 108214 \\
\hline$\# 11$ & Search “neoplasms”[MeSH Terms] & 2609885 \\
\hline$\# 12$ & $\begin{array}{l}\text { Search }\left(\left(\left(\left(\left(\left(\left(\text { cance }{ }^{*}[\text { Title/Abstract] }) \text { OR carcinom* }{ }^{*} \text { Title/Abstract] }\right) \text { OR neoplasm* }{ }^{*} \text { Title/Abstract] }\right) \text { OR }\right.\right.\right.\right.\right. \\
\text { tumo* }[\text { Title/Abstract] }) \text { OR tumou* }[\text { Title/Abstract] }) \text { OR neoplasia[Title/Abstract] }) \text { OR oncolog* }[\text { Title/Abstract] }) \\
\text { OR malignan* [Title/Abstract] }) \text { OR neoplasms[Title/Abstract] }\end{array}$ & 2345516 \\
\hline$\# 13$ & $\# 11$ OR \#12 & 3231222 \\
\hline \#14 & Search “anxiety”[MeSH Terms] & 56178 \\
\hline \#15 & Search “anxiety disorders" [MeSH Terms] & 67844 \\
\hline$\# 16$ & Search “depression” [MeSH Terms] & 153512 \\
\hline \#17 & Search “depressive disorder”[MeSH Terms] & 80897 \\
\hline$\# 18$ & Search “mood disorders" [MeSH Terms] & 115156 \\
\hline$\# 19$ & Search “affective disorders, psychotic"[MeSH Terms] & 33045 \\
\hline \#20 & Search “stress, psychological”[MeSH Terms] & 92744 \\
\hline$\# 21$ & \#14 OR \#15 OR \#16 OR \#17 OR \#18 OR \#19 OR \#20 & 346299 \\
\hline$\# 22$ & $\begin{array}{l}\text { Search }\left(\left(\left(\left(\left(\left(\text { anxiet }{ }^{*}[\text { Title/Abstract] }) \text { OR anxiou* nervousness[Title/Abstract] }\right) \text { OR }\right.\right.\right.\right.\right. \\
\text { hypervigilanc* [Title/Abstract]) OR depression* [Title/Abstract]) OR depressiv* [Title/Abstract]) OR } \\
\left.\left.\text { emotion* }{ }^{*} \text { Title/Abstract] }\right) \text { OR psycholog* [Title/Abstract] }\right) \text { OR affective }{ }^{*}[\text { Title/Abstract] }\end{array}$ & 518732 \\
\hline$\# 23$ & $\# 21 \mathrm{OR} \# 22$ & 687179 \\
\hline$\# 24$ & \#10 AND \#13 AND \#23 & 251 \\
\hline \multicolumn{3}{|c|}{ Cochrane Central Register of Controlled Trials (CENTRAL) } \\
\hline$\# 1$ & MeSH descriptor: [Acupuncture Therapy] explode all trees & 3132 \\
\hline$\# 2$ & MeSH descriptor: [Acupuncture] explode all trees & 151 \\
\hline$\# 3$ & MeSH descriptor: [Acupressure] explode all trees & 227 \\
\hline$\# 4$ & MeSH descriptor: [Acupuncture Points] explode all trees & 1069 \\
\hline$\# 5$ & MeSH descriptor: [Acupuncture, Ear] explode all trees & 123 \\
\hline \#6 & MeSH descriptor: [Auriculotherapy] explode all trees & 129 \\
\hline$\# 7$ & MeSH descriptor: [Electroacupuncture] explode all trees & 473 \\
\hline$\# 8$ & $\begin{array}{l}\text { acupunctur* }^{*} \text { or acupressure }{ }^{*} \text { or auriculotherap }{ }^{*} \text { or electroacupunctur* }{ }^{*} \text { or acupoint }{ }^{*} \text { or needl }{ }^{*} \text { :ti,ab,kw (Word } \\
\text { variations have been searched) }\end{array}$ & 13648 \\
\hline$\# 9$ & $\# 1$ or $\# 2$ or $\# 3$ or $\# 4$ or $\# 5$ or $\# 6$ or $\# 7$ or $\# 8$ & 13710 \\
\hline$\# 10$ & MeSH descriptor: [Neoplasms] explode all trees & 52791 \\
\hline \#11 & $\begin{array}{l}\text { neoplasm }{ }^{*} \text { or tumo* or tumou* or neoplasia or cance* or carcinom* }{ }^{*} \text { or malignan }{ }^{*} \text { or oncolog* :ti,ab,kw (Word } \\
\text { variations have been searched) }\end{array}$ & 94737 \\
\hline$\# 12$ & $\# 10$ or \#11 & 100138 \\
\hline$\# 13$ & MeSH descriptor: [Anxiety] explode all trees & 5187 \\
\hline
\end{tabular}


TABLE 4: Continued.

\begin{tabular}{llr}
\hline ID & Searching strategies & Records \\
\hline$\# 14$ & MeSH descriptor: [Anxiety Disorders] explode all trees & 4954 \\
$\# 15$ & MeSH descriptor: [Depression] explode all trees & 5472 \\
$\# 16$ & MeSH descriptor: [Affective Disorders, Psychotic] explode all trees & 1648 \\
$\# 17$ & MeSH descriptor: [Emotions] explode all trees & 11649 \\
& ${\text { anxiet* }{ }^{*} \text { or anxiou* or nervousness or hypervigilanc* or depression* or depressiv* or emotion* or Psycholog* or }}^{*}$ & 102362 \\
$\# 18$ & affective* :ti,ab,kw (Word variations have been searched) & 103970 \\
$\# 20$ & $\# 13$ or \#14 or \#15 or \#16 or \#17 or \#18 & 108 \\
$\# 21$ & $\# 9$ and \#12 and \#19 & 84 \\
\hline
\end{tabular}

(ii) Acupuncture versus Sham Acupuncture. Sham acupuncture was adopted in two studies $[26,27]$. Due to different types of data, we used descriptive analysis here. One study [26] indicated that there were no significant differences for between-groups comparison in depression $(P=0.442)$, anxiety $(P=0.526)$, and sleep quality $(P=0.557)$. While for within-group comparisons, only true acupuncture group showed a noticeable difference in depression after intervention $(P=0.022)$. For another one [27], effects were similar for both depression and anxiety in within-group comparisons, and also, there were no statistically significant differences between the true acupuncture group and the sham acupuncture group.

3.4.3. Subgroup Analysis on Electroacupuncture. Descriptive analysis was adopted as data synthesis could not be conducted for subgroup analysis on electroacupuncture due to the insufficient number of studies and the inconsistent types of outcome data. Three studies [29, 32, 35] compared electroacupuncture with standard methods of treatment/care and the results were different. One trial [29] with a threearm design indicated that both true electroacupuncture $(P=$ $0.015)$ and sham electroacupuncture $(P=0.0088)$ presented a more effective improvement in HADS-D scores than waitlist control, while, for anxiety, only true intervention was found to be effective $(P=0.044)$. Meanwhile, this study [29] also found no significant improvement in sleep quality (as measured by PSQI) in both true and sham intervention groups, with the $P$ values 0.058 and 0.31 , respectively. However, based on the reported data, we still cannot judge whether true electroacupuncture is superior to the sham approach as between-groups comparisons were not reported in this article [29].

In another study [35], apart from the standard treatment/care in both groups, the intervention group adopted electroacupuncture plus music therapy to compare with the control arm using music therapy only, and the withingroup analysis showed that the HAMD-D scores significantly decreased in both groups after the intervention with the $P$ values, 0.013 and 0.022 , respectively. However, betweengroups comparisons revealed no statistical differences after the intervention $(P=0.431)$. In the study conducted by Frisk et al. [32], subscales of Women's Health Questionnaire (WHQ) and PGWB were employed to evaluate the status of depression and anxiety, and the subscale scores for anxiety (both WHQ and PGWB) were found to be improved significantly in the electroacupuncture group after the treatment, and all sleep parameters (including number of hours slept/night, times of wake-up/night, and WHQ sleep scores) also improved significantly in the electroacupuncture group (within-group comparison).

3.4.4. Subgroup Analysis on Acupressure. There was only one trial [30] adopting acupressure as the intervention approach, and it was found that the acupressure group had lower anxiety and depression scores than the sham acupressure group, but these differences did not reach a significant level.

3.4.5. Subgroup Analysis on Sham Acupoints Stimulation versus Wait-List Control. Comparison between sham acupoints stimulation and wait-list control was only mentioned in one study [29]. In this study, the true electroacupuncture group selected the acupoints located around the joint with the most pain and the acupoints were inserted with needles until receiving "de qi," while the sham comparison used nonpenetrating needles at nonacupuncture and nontrigger points located at least $5 \mathrm{~cm}$ from the joint without evoking the sensation of "de qi." This study indicated that sham electroacupuncture can produce some treatment effects to improve the HADS-D scores in cancer patients $(P=0.0088)$, while, for anxiety and sleep quality, there were no significant differences between groups.

3.5. Adverse Events Associated with Acupoints Stimulation. Six trials [26, 27, 31-34] mentioned the potential adverse events as the safety outcomes, of which three studies [26, 31, 32 ] reported that there were no adverse events associated with acupoints stimulation. In Deng et al's study [27], a total of 11 serious side effects occurred during the intervention such as low blood counts, renal failure, and nausea, but all these negative events were deemed to be not related to acupuncture. The other two studies conducted by Molassiotis et al. [33, 34] monitored adverse events, but the one published in 2012 [33] did not report the details of adverse events in the study results, while the latter one, which was published in 2013 [34], reported that a small number of cases suffered from mild side effects including spot bleeding and minor pain/discomfort. 
None of the included trials assessed the causality between the intervention and the possible adverse events.

\section{Discussion}

In this systematic review, 11 trials with a total of 1073 participants were included. The study findings supported that acupoints stimulation can be a beneficial approach for managing anxiety and depression in cancer patients. However, some methodological flaws were still found in some studies. Considering the potential risks of bias identified in the analyzed trials and the limited number of included trials. The findings of this review should be interpreted prudently, and the current evidence on acupoints stimulation for anxiety and depression in cancer survivors can be rated as suggestive but still not fully conclusive.

Based on the overall analysis, acupoints stimulation as a complementary therapy could improve the mood of depression in patients with malignancy. The possible explanation for it might be that different levels of cortisol, epinephrine, and adrenocorticotropic hormone are associated with the negative moods in cancer patients, which are regulated by HPA and SNS, and acupoints stimulation could positively influence the HPA and SNS based on its potential immunemodulatory effect $[24,25]$. It is noted that significant heterogeneity was identified in the overall assessment, and the possible explanations are as follows. Firstly, various types of acupoints stimulation were used among trials which more or less contribute to the heterogeneity in nature. Secondly, the measures for depression in the analyzed five studies were quite inconsistent, with three trials employing HAMD, and the other two applying HADS-D and BDI-PC, respectively, and the inconsistent outcome assessment tools could be another source for introducing the heterogeneity.

In the subgroup analyses, both manual acupuncture and electroacupuncture showed positive effects on depression when comparing with standard methods of treatment/care, among which two trials compared acupuncture with antidepressant, and one study [28] even demonstrated a more significant effect of acupuncture in relieving depression than using antidepressant. However, conclusion on the superiority of acupuncture to antidepressant should be interpreted with caution because some methodological flaws were identified in the mentioned trial such as the absence of blinding and allocation concealment and unbalanced cointerventions, all of which could increase the risks of bias inevitably.

For anxiety, encouraging results on acupoints stimulation were also reported in corresponding studies. Based on the results of data syntheses as well as descriptive analyses, acupoints stimulation can be a beneficial approach for relieving psychological distresses among oncology patients. However, the evidence concluded from the included studies is not fully conclusive at the current stage as a number of methodological flaws, such as the unclear statement of randomization, blinding and allocation concealment, and unbalanced cointerventions across groups, were identified. It is noted that the studies' effect size would be more inclined to be overestimated when the methodological quality of those studies is unsatisfactory [39].
Our study findings did not reach a conclusion on the evidence of acupoints stimulation for improving sleep quality for the following reasons: conflicting results were reported in the analyzed studies, the number of studies reporting sleep quality was quite limited, and those trials reporting sleep quality only included it as a secondary outcome. Effects of acupoints stimulation on sleep quality in cancer patients should be explored in future studies.

It is noted that several kinds of assessment tools, including CESD, HAMD, SDS, BDI-PC, and HADS, were used in the included studies for measuring anxiety and depression. These tools are generic instruments which can be applied in different populations, and their psychometric properties have also been well documented as valid and reliable measures used in cancer patients. For instance, Cronbach's alpha was greater than 0.75 for CESD [40], HAMD [41], SDS [42], and BDI-PC [43] which indicated a satisfactory internal consistency, and the construct validity of HADS was also tested through the factor analysis [38], and Cronbach's alphas for the anxiety and depression subscales were 0.887 and 0.703 , respectively [44].

Placebo effects of acupoints stimulation should be explored in studies incorporating a true, sham, and usual care group. Based on the study findings, both true and sham acupoints stimulation can relieve anxiety and/or depression more effectively than standard methods of treatment/care, and the true interventions were somewhat better than the sham comparisons although the differences did not reach the statistical significance. Based on these findings, it seems that the treatment effects of acupoints stimulation may be associated with large placebo effects. According to a series of recent randomized controlled trials, placebo effects might partially contribute to the overall therapeutic effects of acupoints stimulation [45-48]. However, specific therapeutic effects of acupoints stimulation still play a crucial role in improving patients' functional status, as a large number of rigorously designed randomized controlled trials and systematic reviews have proved the superiority of true acupuncture/acupressure to the sham comparisons [49-52].

Several reasons could be employed to explain the nonsignificant differences between the true and sham acupoints stimulation in our study findings. Firstly, the nonspecific placebo effects might be exaggerated in the analyzed trials as the outcome measures for anxiety and/or depression were all scales measured by patients themselves or caregivers which can be easily affected by their expectancy to treatment, especially when the blinding design is not well performed. Secondly, it is still uncertain whether the sham procedures used in the included studies are really inert or not. One of the preconditions for developing a sham intervention is that it is indistinguishable from the true treatment but it should be inert in nature, which means having no specific therapeutic effects for the targeted health problem but only producing nonspecific physiological and/or psychological effects (placebo effects) [53]. In the analyzed trials, the design of sham acupoints stimulation might not be very appropriate. For example, nonspecific acupoints (one type of sham modality) which were close to the true acupoints might also produce some treatment effects, because, based on the 
holistic concept of Traditional Chinese Medicine (TCM) theory, acupoints stimulation performs its effects by regulating the whole body functions. The "nonacupoints" described in some trials might be potential, active acupoints which have not been identified on current acupoints chart. All of these designs could generate some specific treatment effects for patients in the sham intervention group. Moreover, in our included trials, only four incorporated a sham intervention arm. The relative small sample size and the methodological flaws identified in those trials made the final data analysis on sham intervention only a preliminary finding and not fully convincing.

Adverse events associated with acupoints stimulation were measured in six studies [26, 27, 31-34] and no serious harm data related to acupoints stimulation were reported. Acupoints stimulation could be a safe intervention used in clinical practice. Other systematic reviews, as well as prospective or retrospective surveys with a large sample size, also supported the idea that acupoints stimulation can be a relatively safe approach $[54,55]$. However, it is noted that some analyzed studies in this review failed to include the safety issue as the outcome measure, and none of the included trials incorporated standardized criteria to assess the causality between the intervention and the reported adverse events.

Our findings suggested that acupoints stimulation can be a promising approach to managing the psychological distresses in cancer patients, but definite evidence still cannot be concluded from this review and the study finding can only be interpreted as suggestive due to the following limitations. Firstly, although the quality of the included studies was generally acceptable, methodological flaws still existed in some trials which could affect the reliability of our findings. Secondly, language bias cannot be excluded as all of the analyzed studies were only English or Chinese publications. In addition, publication bias might exist because there were seldom negative findings reported in our included studies, and we did not perform funnel plot as the number of trials included for each comparison was quite limited (less than ten).

\section{Implications for Future Research and Practice}

This study concluded several implications for future research and practice. Firstly, future research should elaborate more details on the protocols of acupoints stimulation in both true and sham intervention groups including the practitioner, duration of intervention and number of sessions, the selection and location of the true and sham acupoints, and the styles of stimulation. Secondly, apart from the certain psychological measures, some biomarkers [56] such as Interleukin6 (IL-6), Interleukin-1 $\beta$ (IL-1 $\beta$ ), and tumor necrosis factor$\alpha$ (TNF- $\alpha$ ) could be adopted as the potential indicators for emotional problems in future studies, which might minimize the exaggeration of treatment effects to some extent. Moreover, design of sham acupoints stimulation should be more reasonable; sham acupoints should be prudently selected to avoid choosing potentially effective true acupoints. An experienced TCM practitioner could be invited or consulted when developing the intervention protocol and some specialdesigned needles which cannot penetrate the acupoints could be adopted as the sham intervention. Furthermore, standard criteria should be applied to assess the causality between the adverse events and the acupoints stimulation. In addition, clinical researchers should take every effort to minimize the potential risks of bias. Although we understand it is difficult to reach blinding design among research personnel who conduct acupuncture or acupressure, some measures can still be considered to control the potential bias induced by study participants and outcome assessors in clinical settings; for example, researchers could use eye-patches to cover the participants' eyes when providing the intervention, and outcome assessors could be those people who are not involved in any other procedures of the study except for the data collection process $[52,57]$. Finally, future studies should follow the CONSORT [58] and STRICTA [59] guidelines to enhance the report quality of the RCTs on acupoints stimulation.

\section{Conflict of Interests}

The authors declare that there is no conflict of interests regarding the publication of this paper.

\section{Authors' Contribution}

Tao Wang undertook the study design, literature searching, data extraction and analysis, and data interpretation and drafted and revised this paper. Renli Deng supported the study design and revised this paper. Jing-Yu Tan undertook the study design, literature searching, and data extraction and revised this paper. Feng-Guang Guan undertook the study design and revised this paper.

\section{References}

[1] J. Ferlay, I. Soerjomataram, R. Dikshit et al., "Cancer incidence and mortality worldwide: sources, methods and major patterns in GLOBOCAN 2012," International Journal of Cancer, vol. 136, no. 5, pp. E359-E386, 2015.

[2] World Health Organization, "Global health observatory data repository. 2011. Number of deaths (World) by cause," February 2015, http://www.who.int/features/qa/15/en/.

[3] O. Minton, A. Richardson, M. Sharpe, M. Hotopf, and P. Stone, "A systematic review and meta-analysis of the pharmacological treatment of cancer-related fatigue," Journal of the National Cancer Institute, vol. 100, no. 16, pp. 1155-1166, 2008.

[4] E. Dean-Clower, A. M. Doherty-Gilman, A. Keshaviah et al., "Acupuncture as palliative therapy for physical symptoms and quality of life for advanced cancer patients," Integrative Cancer Therapies, vol. 9, no. 2, pp. 158-167, 2010.

[5] M. Hamer, Y. Chida, and G. Y. Molloy, "Psychological distress and cancer mortality," Journal of Psychosomatic Research, vol. 66, no. 3, pp. 255-258, 2009.

[6] K. D. Stein, K. L. Syrjala, and M. A. Andrykowski, "Physical and psychological long-term and late effects of cancer," Cancer, vol. 112, no. 11, supplement, pp. 2577-2592, 2008. 
[7] A. Molassiotis, P. Fernandez-Ortega, D. Pud et al., "Complementary and alternative medicine use in colorectal cancer patients in seven European countries," Complementary Therapies in Medicine, vol. 13, no. 4, pp. 251-257, 2005.

[8] S.-M. Wang, D. Gaal, I. Maranets, A. Caldwell-Andrews, and Z. N. Kain, "Acupressure and preoperative parental anxiety: a pilot study," Anesthesia \& Analgesia, vol. 101, no. 3, pp. 666-669, 2005.

[9] J.-Y. Tan, A. Molassiotis, T. Wang, and L. K. P. Suen, "Current evidence on auricular therapy for chemotherapy-induced nausea and vomiting in cancer patients: a systematic review of randomized controlled trials," Evidence-Based Complementary and Alternative Medicine, vol. 2014, Article ID 430796, 18 pages, 2014.

[10] D. Polsky, J. A. Doshi, S. Marcus et al., "Long-term risk for depressive symptoms after a medical diagnosis," Archives of Internal Medicine, vol. 165, no. 11, pp. 1260-1265, 2005.

[11] W. F. Pirl, "Evidence report on the occurrence, assessment, and treatment of depression in cancer patients," JNCI Monographs, no. 32, pp. 32-39, 2004.

[12] M. Pasquini and M. Biondi, "Depression in cancer patients: a critical review," Clinical Practice \& Epidemiology in Mental Health, vol. 3, no. 3, article 2, 2007.

[13] D. P. H. Stark and A. House, "Anxiety in cancer patients," British Journal of Cancer, vol. 83, no. 10, pp. 1261-1267, 2000.

[14] M. Kangas, J. L. Henry, and R. A. Bryant, "Posttraumatic stress disorder following cancer: a conceptual and empirical review," Clinical Psychology Review, vol. 22, no. 4, pp. 499-524, 2002.

[15] G. P. Zhou, J. Y. Cai, Z. G. Huang, and F. Xu, "Effect of depression on adverse drug reactions from antineoplastics among cancer patients," Chinese Remedies \& Clinics, vol. 9, pp. 916-917, 2009 (Chinese).

[16] E. M. V. Reiche, S. O. V. Nunes, and H. K. Morimoto, "Stress, depression, the immune system, and cancer," The Lancet Oncology, vol. 5, no. 10, pp. 617-625, 2004.

[17] P. B. Jacobsen and H. S. Jim, "Psychosocial interventions for anxiety and depression in adult cancer patients: achievements and challenges," CA: A Cancer Journal for Clinicians, vol. 58, no. 4, pp. 214-230, 2008.

[18] M. M. Lee, S. S. Lin, M. R. Wrensch, S. R. Adler, and D. Eisenberg, "Alternative therapies used by women with breast cancer in four ethnic populations," Journal of the National Cancer Institute, vol. 92, no. 1, pp. 42-47, 2000.

[19] World Health Organization, "Acupuncture: review and analysis of reports on controlled clinical trials," Parkinsonism \& Related Disorders, supplement 2, p. S163, 2002.

[20] C. A. Smith, P. P. J. Hay, and H. MacPherson, "Acupuncture for depression," The Cochrane Database of Systematic Reviews, no. 1, Article ID CD004046, 2010.

[21] S. Horiuchi, A. Tsuda, Y. Honda, H. Kobayashi, M. Naruse, and A. Tsuchiyagaito, "Mood changes by self-administered acupressure in Japanese college students: a randomized controlled trial," Global Journal of Health Science, vol. 7, no. 4, pp. 40-44, 2014.

[22] D. W. H. Au, H. W. H. Tsang, P. P. M. Ling, C. H. T. Leung, P. K. Ip, and W. M. Cheung, "Effects of acupressure on anxiety: a systematic review and meta-analysis," Acupuncture in Medicine, vol. 33, no. 5, pp. 353-359, 2015.

[23] Y. Y. Chan, W. Y. Lo, S. N. Yang, Y. H. Chen, and J. G. Lin, "The benefit of combined acupuncture and antidepressant medication for depression: a systematic review and metaanalysis," Journal of Affective Disorders, vol. 176, pp. 106-117, 2015.
[24] H. J. Park, H. J. Park, Y. Chae, J. W. Kim, H. Lee, and J.H. Chung, "Effect of acupuncture on hypothalamic-pituitaryadrenal system in maternal separation rats," Cellular and Molecular Neurobiology, vol. 31, no. 8, pp. 1123-1127, 2014.

[25] L. M. Thornton, B. L. Andersen, and W. P. Blakely, “The pain, depression, and fatigue symptom cluster in advanced breast cancer: covariation with the hypothalamic-pituitary-adrenal axis and the sympathetic nervous system," Health Psychology, vol. 29, no. 3, pp. 333-337, 2010.

[26] T. Bao, L. Cai, C. Snyder et al., "Patient-reported outcomes in women with breast cancer enrolled in a dual-center, doubleblind, randomized controlled trial assessing the effect of acupuncture in reducing aromatase inhibitor-induced musculoskeletal symptoms," Cancer, vol. 120, no. 3, pp. 381-389, 2014.

[27] G. Deng, Y. Chan, D. Sjoberg et al., "Acupuncture for the treatment of post-chemotherapy chronic fatigue: a randomized, blinded, sham-controlled trial," Supportive Care in Cancer, vol. 21, no. 6, pp. 1735-1741, 2013.

[28] Y. Feng, X.-Y. Wang, S.-D. Li et al., "Clinical research of acupuncture on malignant tumor patients for improving depression and sleep quality," Journal of Traditional Chinese Medicine, vol. 31, no. 3, pp. 199-202, 2011.

[29] J. J. Mao, J. T. Farrar, D. Bruner et al., "Electroacupuncture for fatigue, sleep, and psychological distress in breast cancer patients with aromatase inhibitor-related arthralgia: a randomized trial," Cancer, vol. 120, no. 23, pp. 3744-3751, 2014.

[30] W.-R. Tang, W.-J. Chen, C.-T. Yu et al., "Effects of acupressure on fatigue of lung cancer patients undergoing chemotherapy: an experimental pilot study," Complementary Therapies in Medicine, vol. 22, no. 4, pp. 581-591, 2014.

[31] E. M. Walker, A. I. Rodriguez, B. Kohn et al., "Acupuncture versus venlafaxine for the management of vasomotor symptoms in patients with hormone receptor-positive breast cancer: a randomized controlled trial," Journal of Clinical Oncology, vol. 28, no. 4, pp. 634-640, 2010.

[32] J. Frisk, A.-C. Källström, N. Wall, M. Fredrikson, and M. Hammar, "Acupuncture improves health-related quality-of-life (HRQoL) and sleep in women with breast cancer and hot flushes," Supportive Care in Cancer, vol. 20, no. 4, pp. 715-724, 2012.

[33] A. Molassiotis, J. Bardy, J. Finnegan-John et al., "Acupuncture for cancer-related fatigue in patients with breast cancer: a pragmatic randomized controlled trial," Journal of Clinical Oncology, vol. 30, no. 36, pp. 4470-4476, 2012.

[34] A. Molassiotis, J. Bardy, J. Finnegan-John et al., "A randomized, controlled trial of acupuncture self-needling as maintenance therapy for cancer-related fatigue after therapist-delivered acupuncture," Annals of Oncology, vol. 24, no. 6, pp. 1645-1652, 2013.

[35] C. Y. Xiang, Q. Guo, J. Liao, S. G. Wang, Y. F. Yang, and Y. H. Feng, "Effect of therapy of traditional Chinese medicine five element music and electroacupuncture on the depression levels of cancer patients," Chinese Journal of Nursing, vol. 41, no. 11, pp. 969-972, 2006.

[36] M. Q. Shi, K. Han, and Y. L. Xia, "Study about soothing liver and reinforcing essence of kidney by acupuncture in adjusting depression of gynecological malignancies after surgery and chemotherapy," Journal of Liaoning University of TCM, vol. 15, no. 2, pp. 164-168, 2013.

[37] A. D. Furlan, A. Malmivaara, R. Chou et al., "2015 Updated method guideline for systematic reviews in the Cochrane back and neck group," Spine, vol. 40, no. 21, pp. 1660-1673, 2015. 
[38] A. B. Smith, P. J. Selby, G. Velikova et al., "Factor analysis of the hospital anxiety and depression scale from a large cancer population," Psychology and Psychotherapy: Theory, Research and Practice, vol. 75, no. 2, pp. 165-176, 2002.

[39] R. Kunz, G. Vist, and A. D. Oxman, "Randomisation to protect against selection bias in healthcare trials," The Cochrane Database of Systematic Reviews, no. 2, Article ID MR000012, 2007.

[40] M. J. Schroevers, R. Sanderman, E. van Sonderen, and A. V. Ranchor, "The evaluation of the Center for Epidemiologic Studies Depression (CES-D) scale: depressed and positive affect in cancer patients and healthy reference subjects," Quality of Life Research, vol. 9, no. 9, pp. 1015-1029, 2000.

[41] M. Olden, B. Rosenfeld, H. Pessin, and W. Breitbart, "Measuring depression at the end of life: is the hamilton depression rating scale a Valid instrument?" Assessment, vol. 16, no. 1, pp. 43-54, 2009.

[42] W. Dugan, M. V. McDonald, S. D. Passik, B. D. Rosenfeld, D. Theobald, and S. Edgerton, "Use of the Zung self-rating depression scale in cancer patients: feasibility as a screening tool," Psycho-Oncology, vol. 7, no. 6, pp. 483-493, 1998.

[43] K. Mystakidou, E. Tsilika, E. Parpa, V. Smyrniotis, A. Galanos, and L. Vlahos, "Beck depression inventory: exploring its psychometric properties in a palliative care population of advanced cancer patients," European Journal of Cancer Care, vol. 16, no. 3, pp. 244-250, 2007.

[44] K. Mystakidou, E. Tsilika, E. Parpa, E. Katsouda, A. Galanos, and L. Vlahos, "The Hospital Anxiety and Depression Scale in Greek cancer patients: psychometric analyses and applicability," Supportive Care in Cancer, vol. 12, no. 12, pp. 821-825, 2004.

[45] K. Linde, K. Niemann, A. Schneider, and K. Meissner, "How large are the nonspecific effects of acupuncture? A metaanalysis of randomized controlled trials," BMC Medicine, vol. 8, article 75, 2010.

[46] Y. H. Koog, S. R. We, and B.-I. Min, "Three-armed trials including placebo and no-treatment groups may be subject to publication bias: systematic review," PLoS ONE, vol. 6, no. 5, Article ID e20679, 2011.

[47] S. R. We, Y. H. Koog, M. S. Park, and B.-I. Min, "Placebo effect was influenced by publication year in three-armed acupuncture trials," Complementary Therapies in Medicine, vol. 20, no. 1-2, pp. 83-92, 2012.

[48] Y. H. Koog and W. Y. Jung, "Time course of placebo effect of acupuncture on pain: a systematic review," ISRN Pain, vol. 2013, Article ID 204108, 7 pages, 2013.

[49] A. F. Molsberger, T. Schneider, H. Gotthardt, and A. Drabik, "German randomized acupuncture trial for chronic shoulder pain (GRASP) - a pragmatic, controlled, patient-blinded, multi-centre trial in an outpatient care environment," Pain, vol. 151, no. 1, pp. 146-154, 2010.

[50] K. Itoh, Y. Katsumi, S. Hirota, and H. Kitakoji, "Effects of trigger point acupuncture on chronic low back pain in elderly patients-a sham-controlled randomised trial," Acupuncture in Medicine, vol. 24, no. 1, pp. 5-12, 2006.

[51] Y. Sun and T. J. Gan, "Acupuncture for the management of chronic headache: a systematic review," Anesthesia and Analgesia, vol. 107, no. 6, pp. 2038-2047, 2008.

[52] J. Y. Tan, L. K. Suen, T. Wang, and A. Molassiotis, "Sham acupressure controls used in randomized controlled trials: a systematic review and critique," PLoS ONE, vol. 10, no. 7, Article ID e0132989, 2015.
[53] I. Lund, J. Näslund, and T. Lundeberg, "Minimal acupuncture is not a valid placebo control in randomised controlled trials of acupuncture: a physiologist's perspective," Chinese Medicine, vol. 4, article 1, 2009.

[54] S. Xu, L. Wang, E. Cooper et al., "Adverse events of acupuncture: a systematic review of case reports," Evidence-Based Complementary and Alternative Medicine, vol. 2013, Article ID 581203, 15 pages, 2013.

[55] J.-E. Park, M. S. Lee, J.-Y. Choi, B.-Y. Kim, and S.-M. Choi, "Adverse events associated with acupuncture: a prospective survey," The Journal of Alternative and Complementary Medicine, vol. 16, no. 9, pp. 959-963, 2010.

[56] C. X. Liu, L. L. Han, Z. Z. Yang, and Q. J. Yuan, "An analysis of serum levels of cytokines and relative factors in depression patients," Chinese Journal of Behavioral Medicine \& Brain Science, vol. 23, no. 9, pp. 801-804, 2014.

[57] D. Kondziolka, T. Lemley, J. R. W. Kestle, L. D. Lunsford, G. H. Fromm, and P. J. Jannetta, "The effect of single-application topical ophthalmic anesthesia in patients with trigeminal neuralgia: a randomized double-blind placebo-controlled trial," Journal of Neurosurgery, vol. 80, no. 6, pp. 993-997, 1994.

[58] K. F. Schulz, D. G. Altman, and D. Moher, "CONSORT 2010 statement: updated guidelines for reporting parallel group randomised trials," BMC Medicine, vol. 8, no. 1, article 18, 2010.

[59] H. MacPherson, D. G. Altman, R. Hammerschlag et al., "Revised standards for reporting interventions in clinical trials of acupuncture (STRICTA): extending the CONSORT statement," Journal of Evidence-Based Medicine, vol. 3, no. 3, pp. 140$155,2010$. 


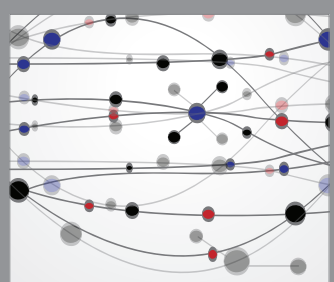

The Scientific World Journal
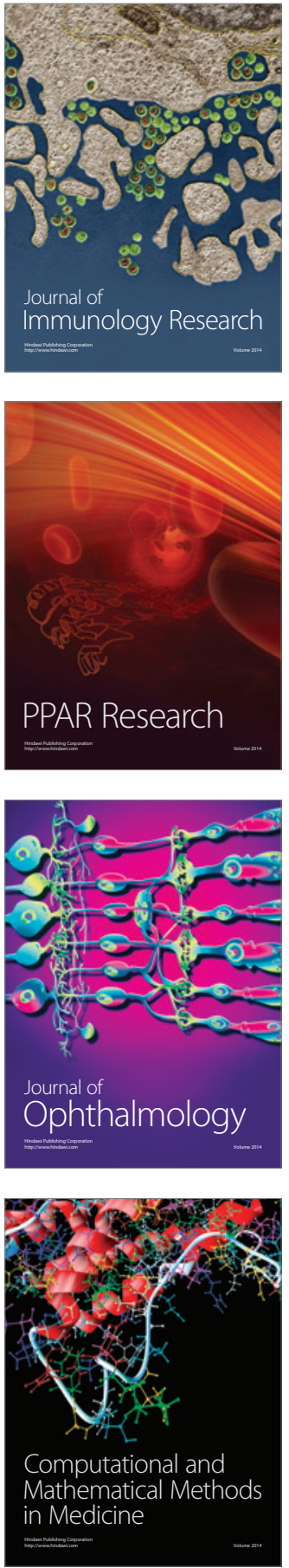

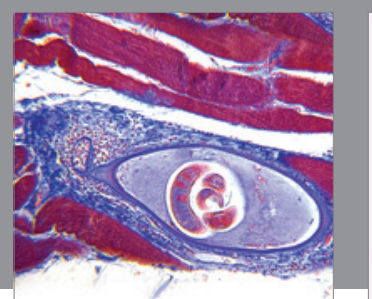

Gastroenterology Research and Practice

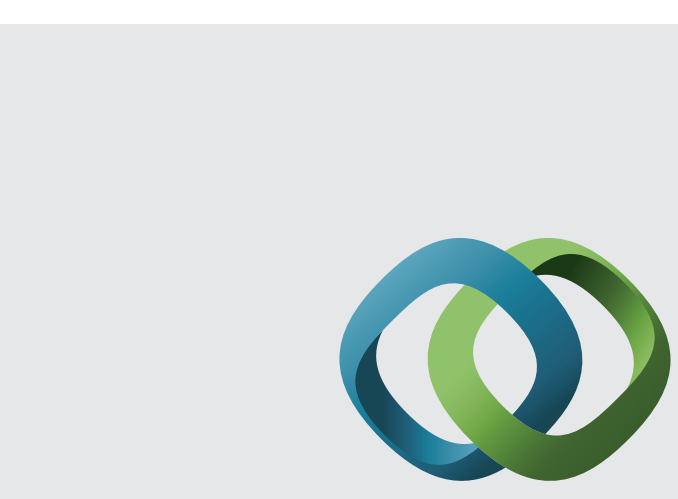

\section{Hindawi}

Submit your manuscripts at

http://www.hindawi.com
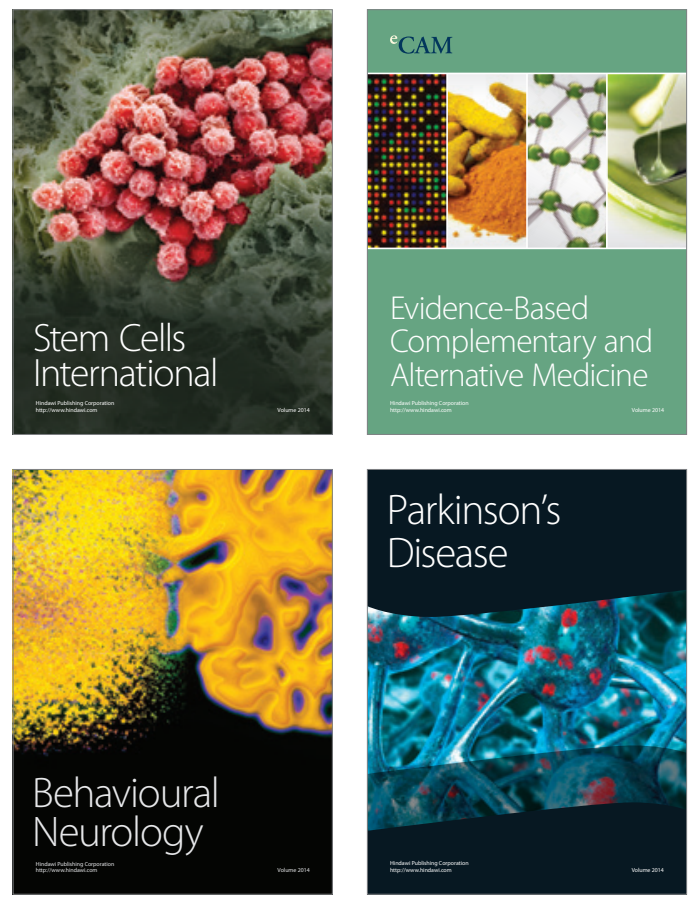
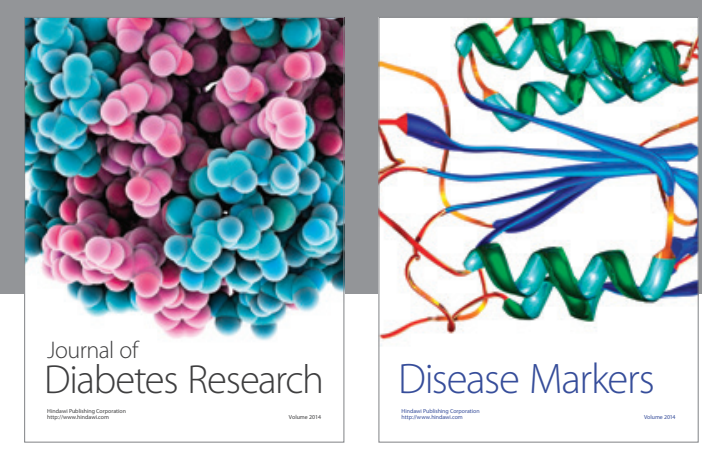

Disease Markers
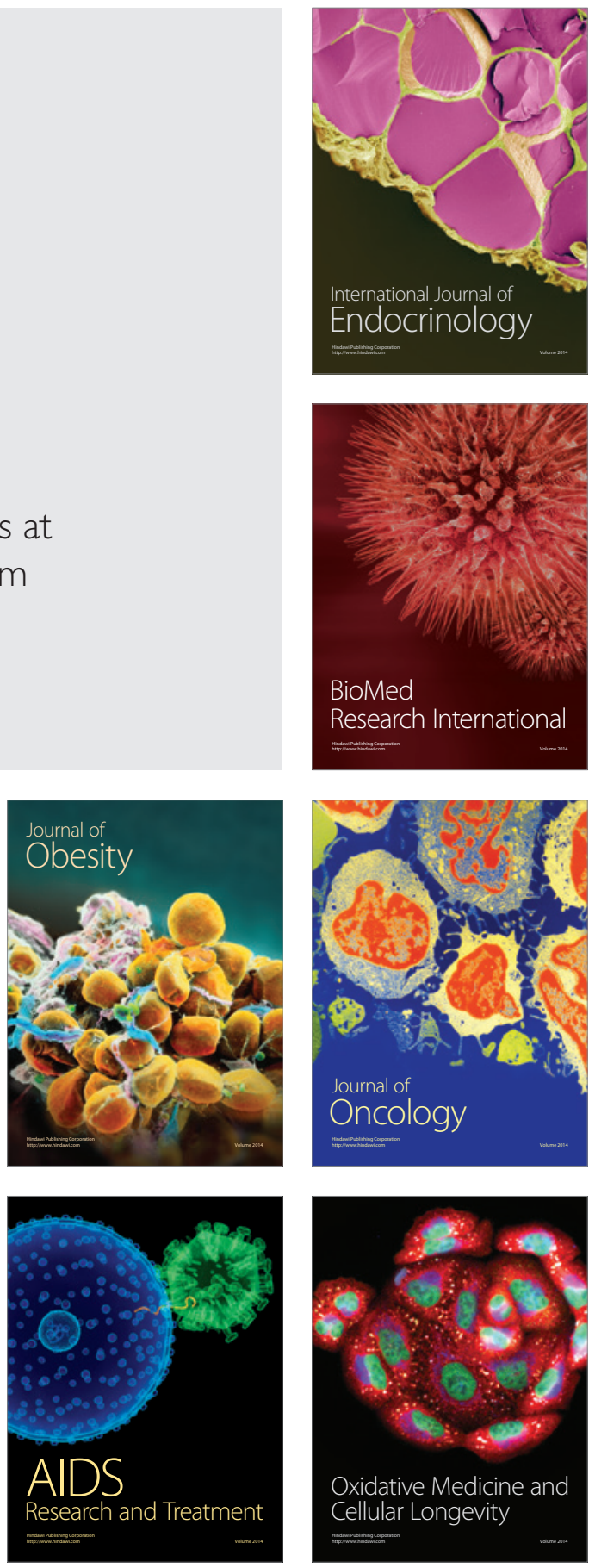